\title{
Combining physical training with transcranial direct current stimulation to improve gait in Parkinson's disease: a pilot randomised controlled study
}

\section{Introduction}

Parkinson's disease is a common cause of gait and postural instability, which may not respond to levodopa (1). Its treatment is therefore therapeutically challenging. Given the reduced activity in premotor and primary motor cortical regions in Parkinson's disease (2), these areas may be important therapeutic non-invasive neurostimulatory targets for patients with Parkinson's disease and gait disturbance (3).

Physical training appears to induce physiological changes in the primary motor cortex of patients with Parkinson's disease (4). Non-invasive brain stimulation techniques such as transcranial direct current stimulation (tDCS) has also been shown to improve motor learning and rehabilitation in Parkinson's disease (5), and other primary gait disorders (6), although outcomes have been mixed (7).

The objective of this study was to improve gait velocity (primary gait outcome) and the response on the pull test (primary balance outcome) in patients with Parkinson's disease by combining tDCS with physical training. We hypothesised that anodal tDCS time-locked to physical training would improve gait and balance in Parkinson's disease above and beyond the effects of tDCS in isolation. Our primary gait outcome was gait velocity as this is a functional marker of disease severity, and our primary balance outcome was recovery of stance in the pull test (8), postural instability being a common cause of falls in these patients.

\section{METHODS}

\section{Trial design}

Patients were assigned to one of two groups (Figure 1: 'physical-training' [n=8] or 'No physicaltraining' [n=8]) using an online randomisation software tool (Research Randomizer, Version 3.0). Patients in Group I received tDCS during a fifteen-minute period of physical training, as part of a nested study. Patients in Group II received only tDCS, without physical training. For both groups, the 
application of tDCS was also randomised to either real tDCS stimulation or sham stimulation in the first session. All subjects returned 1 week later, to allow an appropriate wash-out period for the neurostimulation. In this second session subjects in the 'physical training' group again received physical training, but those that received real stimulation in the first session were given sham stimulation, and vice versa (Figure 1). Patients in the 'No physical training' group again received only neurostimulation in this second session, with those that received real stimulation in the first session given sham stimulation, and vice versa. Outcomes were recorded prior to any intervention (baseline), and after intervention (final assessment). Patients were assigned to three clinicians experienced in the application of both physical therapy and neurostimulation.

\section{Participants}

Forty consecutive patients fulfilling the UK Society Brain Bank criteria for idiopathic Parkinson disease (9) were approached for this study, within the allocated recruitment period of 1 year (Figure 1). Written informed consent was obtained for all participants. Patients with severe freezing, daily falls, dementia (mini-mental state examination score (10) less than 24/30), or other disorders affecting gait and balance were excluded $(n=14)$. All patients underwent magnetic resonance imaging of the brain to exclude alternative central nervous system pathologies that could affect gait. Each subject was tested whilst on medication, and assessments carried out at the same time of the day in the first and second session to avoid diurnal variation and on/off medication effects.

The study was approved by the local ethics committee, and conducted in accordance to the Declaration of Helsinki.

\section{Interventions}

\section{Physical training}

Our physical training protocol (Appendix 1) lasted 15 minutes and focused on improving gait initiation, stride length, gait velocity, arm swing, and balance. To stand from sitting, patients were instructed to shift their body forwards towards the edge of the seat, place the heels firmly on the 
ground, as far back underneath the chair as possible, and move the centre of gravity forwards, to stand in a arc-like fashion forwards and upwards. They were instructed to walk taking long strides with adequate floor clearance, and swing the arms to maintain balance. For the turning practice, they were asked to use whole body movements, taking steps around objects to avoid rapidly switching direction. For balance practice patients were asked to pick a book from the floor using both hands, at increasing distance, by taking a forward step. They were also given backward pulls and instructed to take anticipatory steps to maintain balance when the pressure on the shoulders was felt. The protocol was designed by our local physiotherapists based on published practice recommendations (11).

\section{Transcranial direct current stimulation (tDCS)}

For the tDCS, we used an identical montage and stimulation protocol as described in Kaski et al., (12). Briefly, a direct current rectangular stimulating (anodal) electrode $(10 \mathrm{x} 4 \mathrm{~cm})$ was placed centrally across the scalp to cover a region $10-20 \%$ anterior to $\mathrm{Cz}$ as measured from the midline of the electrode. A reference electrode $(4 \mathrm{x} 4 \mathrm{~cm})$ was placed at the inion. A $2 \mathrm{~mA}$ current was delivered for 15 minutes during training ('physical training' group), or during the execution of the git and balance tasks ('No physical training' group). Electrodes were secured onto the scalp during the assessments using a modified diving head cap, and tDCS battery fitted on a lightweight backpack.

\section{Outcome measures}

The primary outcome for gait was gait velocity. Secondary outcomes included stride length, $3 \mathrm{~m}$ 'Timed Up and Go', 6m walk. The primary balance outcome was a quantitative pull test. All tests were recorded on video camera (Veho Kuzo HD camcorder) to assess gait velocity, stride length, and stride length variability. Observational assessments of gait have been previously validated in the clinical setting (13).

For gait assessments, subjects were instructed to "walk as quickly, but safely, as possible". The duration of walking during the middle $4 \mathrm{~m}$ of the $6 \mathrm{~m}$ walk was used to calculate mean gait velocity $(\mathrm{m} / \mathrm{s})$, to avoid variability from acceleration/deceleration effects. Stride length (distance from the initial contact of one foot with the ground to the following contact of the same foot) was measured 
against floor markers $15 \mathrm{~cm}$ apart, using frame-by-frame video analysis. Mean gait velocity for the $6 \mathrm{~m}$ walk obtained from the Swaystar (see below) was cross-referenced with the video data acquisition for validation.

For the Timed 'Up and Go', subjects began seated in an armless office chair and were asked to get up, walk 3 metres, turn round, return to the chair and sit down again. A verbal "go" signal was given to start the test. Subjects were asked to perform the task at a brisk but safe pace. The total duration of the task was calculated from the moment the subject began to get up until they were comfortable on the chair again. The 'turn' phase of the Timed 'Up and Go' was also analysed separately, using the Swaystar system. The onset of the turn was marked by a change in the polarity of the Swaystar angular displacement signal. The end of the turn was taken as the end of this polarity change, as the signal reached a plateau, before gait recommenced.

The pull test is a clinical tool used to evaluate postural instability in patients with Parkinson's disease (14). Subjects stood in a comfortable stance position with feet at shoulder width, and eyes open. They were warned that they would receive a sudden and firm shoulder pull, delivered by the examiner standing directly behind the subject. The examiner then delivered a single pull using sufficient strength in order to force subjects to take at least one corrective step backwards. The test was repeated a total of 3 times. The same examiner (DK) performed the pull test on every subject to reduce the pull strength variability. The shoulder pull forces were not quantitatively controlled but recordings showed that subjects' backward trunk velocity was approximately equal (mean backward velocity $=$ $32.5 \mathrm{deg} / \mathrm{s}, \mathrm{SD}=5.8$ ). Trunk sway recordings were started just prior to the pull and terminated 5 seconds later, or until loss of balance occurred. Subjects were prevented from falling by the examiner and an assistant standing next to the subject. Angular trunk displacement and velocity was recorded for subjects in the pitch (sagittal) plane (SwayStar System, Balance Int. Innovations GmbH, Switzerland (15)) to provide a quantitative measure of the pull test in all subjects. Thus, for the recorded (angular trunk movements) version of the pull test we report the time taken to regain a trunk angular position within 2 degrees of the baseline (quiet standing), which is easy to measure objectively (Figure 2A) and correlates well with the number of falls in the preceding year $(\mathrm{r}=0.8$, 
$\mathrm{p}=0.0002$; Figure 2B), suggesting it is a clinically relevant measure of imbalance. We also recorded the peak pull velocity and displacement (to ascertain stimulus consistency) and peak recovery phase velocity within the first second following maximum pitch backward displacement.

\section{Randomisation and blinding}

Randomisation was performed by the unblinded researcher by entering the anonymised patient details into the software prior to the patient's arrival. This generated a code relating to the intervention arm (physical training versus no training), and stimulation type (real versus sham). Data were analysed blindly, without knowledge of whether patients received real or sham stimulation.

\section{Data analysis}

Video and SwayStar data were acquired simultaneously for all tasks. For the video data, the first three and the last two steps of each gait cycle were eliminated from the data set to remove acceleration and deceleration effects. Stride length was defined as the distance from the initial contact of one foot with the ground to the following contact of the same foot.

\section{Statistical methods}

Statistical analysis was carried out using SPSS 18.0 with significance set at $p<0.05$ (two-tailed), and consisted of paired student t-tests. Where Bonferroni correction was applied for multiple comparisons, the adjusted significance level is reported in the text.

For individual correlations, correlation coefficients were compared using Fisher's r-to-z transformation. $\mathrm{z}$ values were obtained using the formula $\mathrm{z}_{\mathrm{r}}=(1 / 2)[\log \mathrm{e}(1+\mathrm{r})-\log \mathrm{e}(1-\mathrm{r})]$ where $\mathrm{r}$ is the regression coefficient. A line was fitted through the data set using the method of least squares, and a z-test performed to calculate the $\mathrm{p}$ value.

To assess the effect of combining tDCS with physical training we compared the average \% change in performance in patients receiving real stimulation in Group I vs real stimulation in Group II. We compared the average $\%$ change in performance in patients receiving real vs sham stimulation in 
Group II to assess the isolated effect of tDCS stimulation (independent of physical training). To assess the isolated effect of physical training (independent of tDCS) we compared the average $\%$ change in performance in patients receiving sham in Group I vs patients receiving sham stimulation in Group II. Where an isolated effect of physical training was observed, we compared the average $\%$ change in performance in patients receiving real stimulation in Group I vs sham stimulation in Group I, to assess the effect of combining tDCS with physical training - above and beyond the effect of physical training alone.

\section{Results}

Ten patients declined participation, and sixteen patients were enrolled.

Table 1 shows the averaged percentage change for all groups and tasks, whilst absolute values for baseline and final assessments for all tasks are shown in Table 2. Statistical results are reported in the text.

\section{Gait velocity}

The combination of tDCS+physical training significantly increased gait velocity compared to tDCS alone $(\mathrm{p}<0.001)$. There was an isolated effect of physical training $(\mathrm{p}=0.03)$ but no overall effect of tDCS alone $(\mathrm{p}=0.12)$. Percentage increase in gait velocity was greater for patients receiving a combination of $\mathrm{tDCS}+$ physical training than with physical training alone. In the tDCS+physical training group, gait velocity correlated with UPDRS $(\mathrm{r}=0.77, \mathrm{p}=0.025)$ but not with age, leukoaraiosis (Fazekas score) or mini-mental state examination ( $\mathrm{p}>0.1$ for all).

\section{Stride length}

Stride length increased between baseline and final assessments with combined tDCS+physical training, compared to tDCS alone ( $\mathrm{p}=0.01)$. There was no isolated effect of stimulation $(\mathrm{p}=0.33)$, but physical training alone significantly increased stride length $(\mathrm{p}=0.03)$. The effects of tDCS+physical training were nevertheless greater than physical training alone $(\mathrm{p}=0.03)$. 


\section{Timed Up and Go}

There was no difference in baseline timed ' $U p$ and Go' duration between real and sham stimulation groups (paired t-test; $\mathrm{p}=0.72$ for the 'physical training' group, and $\mathrm{p}=0.85$ for 'No-physical training' group). tDCS+physical training resulted in significant improvements for timed 'Up and Go' duration compared to tDCS alone $(\mathrm{P}=0.04)$. There was no isolated effect of $\mathrm{tDCS}(\mathrm{p}=0.52)$ nor physical training ( $\mathrm{p}=0.31$ ). Sub-analysis of the turn phase of the timed ' $U p$ and Go' also revealed significant improvements with tDCS+physical training, but not with tDCS or physical training alone.

6 metre walk

tDCS+physical training decrease walking time compared to tDCS alone $(\mathrm{p}=0.01)$ but $\mathrm{tDCS}$ had no effect in isolation $(\mathrm{p}=0.48)$. There was a significant effect physical training alone $(\mathrm{p}=0.042)$, but this was comparatively less than with tDCS+physical training $(\mathrm{p}=0.05)$.

\section{Pull test}

tDCS+physical training reduced the time taken to regain stability following the retropulsion stimulus (Figure 2A) compared to tDCS alone ( $\mathrm{p}=0.01)$. There was no isolated effect of stimulation $(\mathrm{p}=0.94)$ or physical training $(\mathrm{p}=0.87)$.

\section{Individual correlations}

In the tDCS+physical training group there was a positive correlation between disease severity and \% reduction in walking time (timed 'Up and Go' vs. United Parkinson's Disease Rating Scale; $r=0.74, p=0.03$; timed 'Up and Go' vs. Hoehn-Yahr stage; $r=0.62, p=0.05$ ) but not with age (United Parkinson's Disease Rating Scale vs. age $r=0.26, p=0.53$ ), white matter disease (United Parkinson's Disease Rating Scale vs. Fazekas r=0.005, p=0.99), or cognitive impairment (United Parkinson's Disease Rating Scale vs. Mini-mental State Examination $\mathrm{r}=0.24, \mathrm{p}=0.57)$. We performed post hoc analyses on 'responders' in the tDCS+Physical training group that had a moderate effect size (Cohen's $\delta>0.5)$ in the timed 'Up and Go' and 
$6 \mathrm{~m}$ walk (n=6). Improvements in gait were strongly correlated with the intensity of the dopaminergic treatment (timed 'Up and Go' vs. levodopa daily equivalent dose $r=0.83$, $\mathrm{p}=0.02 ; 6 \mathrm{~m}$ walk vs. levodopa daily equivalent dose $\mathrm{r}=0.82, \mathrm{p}=0.02)$. Additionally, there was a significant correlation between non-motor symptoms and improvements in gait (timed 'Up and Go' vs. United Parkinson's Disease Rating Scale I: r=0.71, $\mathrm{p}=0.05 ; 6 \mathrm{~m}$ walk vs. United Parkinson's Disease Rating Scale I r=0.85, $\mathrm{p}=0.01$ ).

\section{Discussion}

This double-blind, randomised, sham-controlled pilot study evaluated bihemispheric anodal tDCS with physical training as a 'proof-of-principle' treatment for gait and balance disturbance in Parkinson's disease. We found a significant benefit of combining tDCS with physical training for gait velocity (primary gait outcome) and the performance on the pull test (primary balance outcome), but no isolated effect of tDCS or physical training.

Gait and balance impairment in patients with Parkinson's disease have proved notoriously difficult to treat, and although some evidence suggests that freezing of gait may improve with levodopa (16), one study indicated that gait may worsen in medicated patients, perhaps as a result of drug-induced dyskinesia (17). As a result, there has been a drive towards non-pharmacological therapies. Deep brain stimulation has revolutionised the management of dopaminergic motor features in many patients with severe Parkinson's disease, and whilst there is some evidence in support for in the treatment of gait disorders in these patients (18), it's effect on the non-dopaminergic motor features of Parkinson's disease, such as gait and balance has been disappointing. Non-invasive treatment options, in particular repetitive transcranial magnetic stimulation showed early promise in the treatment of gait in this group of patients $(19,20)$, but subsequent studies using intermittent magnetic stimulation (21) and tDCS (22) have yielded negative results. One study assessed the effect of tDCS when applied concomitantly with gait rehabilitation in patients with chronic stroke finding no additional benefit over and above rehabilitation alone (23). In contrast, tDCS applied during physical gait and balance training improved gait velocity and balance in patients with small vessel disease (6). 
Combining physical gait and balance training with tDCS over primary motor and premotor cortex has a physiological basis - physical training in Parkinson's disease normalises cortical excitability in M1 (4), whilst tDCS may lower the threshold for these changes to occur. Given the bilateral representation of cortical gait regions, one might expect improvements in gait to require simultaneous modulation of cortical activity bilaterally. Cortical leg stimulation using non-invasive techniques is more challenging than upper limb cortical stimulation given its deeper anatomical location, and more vertical orientation than the hand motor cortex. The electrode montage used in the present study was previously shown to alter cortical excitability in both lower limbs (12), but not hand areas, with a sufficiently high current to penetrate deep into the medial surface of the cortex (24), rather than the cortical convexity (12). Our anodal electrode covered a surface overlying primary motor and premotor areas but we cannot comment on possible differential stimulatory effects over these respective cortical regions.

Patients with more severe motor symptoms (United Parkinson's Disease Rating Scale III and Hoehn Yahr scale) tended to show an improvement in walking time (primary outcome) with tDCS compared to patients with milder disease. Although the number of patients tested is insufficient to draw firm conclusions from a correlation analysis, our data suggest that patients with more advanced disease are more likely to benefit from combined direct current stimulation and physical training than patients with mild disease. In contrast, individual gait outcomes did not correlate with age, the degree of leukoaraiosis, or cognitive impairment, suggesting that individual patients at the extremes of the disease process may be less likely to respond to neurostimulation. We cannot comment on whether disease severity or dopaminergic treatment dose was responsible for the improvements in gait apparent in this small number of individual patients. Our results suggest that further therapeutic studies combining direct current stimulation and dopaminergic medications are warranted.

There are a number of limitations of this study. Firstly, whilst our patient sample size is adequate for a pilot proof-of-principle study, it lacks the power to draw conclusive results regarding the combination of physical training and tDCS to improve gait in patients with 
Parkinson's disease. Based on the observed effect size in this pilot study, a power calculation revealed that at least 14 patients per treatment arm $(\alpha=0.05$; power $=0.8)$ should be recruited for a definitive trial. Secondly, the lack of follow-up assessments meant that we were unable to comment on possible longer-term benefit of the interventions. Finally, we recognise that there may have been the potential for selection bias given that we sought to include only patients with Parkinson's disease with a primary complaint of a gait disorder. This was however a necessary selection process to explore the effect of tDCS and physical training on gait.

In conclusion, we have shown that the combination of anodal tDCS placed centrally over the primary motor and premotor cortices of both hemispheres improves gait velocity and balance in patients with Parkinson's disease. Our results suggest that clinical rehabilitation of gait in these patients could be combined with tDCS to enhance the effects of therapy.

\section{Clinical Messages}

Combining non-invasive brain stimulation to the motor cortex during physical training may improve gait and balance in patients with Parkinson's disease.

Power calculations revealed that 14 patients per treatment arm $(\alpha=0.05$; power $=0.8)$ are required for a definitive trial.

Better outcomes were observed in patients with more advanced disease.

Our results suggest that clinical rehabilitation of gait in these patients could be combined with tDCS to enhance the effects of therapy.

\section{References}


1. Hely MA, Morris JG, Reid WG, Trafficante R. Sydney Multicenter Study of Parkinson's disease: non-L-dopa-responsive problems dominate at 15 years. Mov Disord. 2005;20(2):190-9. Epub 2004/11/20.

2. Playford ED, Jenkins IH, Passingham RE, Nutt J, Frackowiak RS, Brooks DJ. Impaired mesial frontal and putamen activation in Parkinson's disease: a positron emission tomography study. Ann Neurol. 1992;32(2):151-61. Epub 1992/08/01.

3. Pascual-Leone A, Valls-Sole J, Brasil-Neto JP, Cammarota A, Grafman J, Hallett M. Akinesia in Parkinson's disease. II. Effects of subthreshold repetitive transcranial motor cortex stimulation. Neurology. 1994;44(5):892-8. Epub 1994/05/01.

4. Fisher BE, Wu AD, Salem GJ, Song J, Lin CH, Yip J, et al. The effect of exercise training in improving motor performance and corticomotor excitability in people with early Parkinson's disease. Archives of physical medicine and rehabilitation. 2008;89(7):1221-9. Epub 2008/06/07.

5. Williams JA, Imamura M, Fregni F. Updates on the use of non-invasive brain stimulation in physical and rehabilitation medicine. J Rehabil Med. 2009;41(5):305-11. Epub 2009/04/14.

6. Kaski D, Dominguez RO, Allum JH, Bronstein AM. Improving Gait and Balance in Patients With Leukoaraiosis Using Transcranial Direct Current Stimulation and Physical Training: An Exploratory Study. Neurorehabil Neural Repair. 2013. Epub 2013/07/31.

7. Alon G, Yungher DA, Shulman LM, Rogers MW. Safety and immediate effect of noninvasive transcranial pulsed current stimulation on gait and balance in Parkinson disease. Neurorehabil Neural Repair. 2012;26(9):1089-95. Epub 2012/05/15.

8. Hunt AL, Sethi KD. The pull test: a history. Mov Disord. 2006;21(7):894-9. Epub 2006/05/11.

9. Hughes AJ, Daniel SE, Kilford L, Lees AJ. Accuracy of clinical diagnosis of idiopathic Parkinson's disease: a clinico-pathological study of 100 cases. J Neurol Neurosurg Psychiatry. 1992;55(3):181-4. Epub 1992/03/01.

10. Folstein MF, Folstein SE, McHugh PR. "Mini-mental state". A practical method for grading the cognitive state of patients for the clinician. J Psychiatr Res. 1975;12(3):189-98. Epub 1975/11/01.

11. Suchowersky O, Gronseth G, Perlmutter J, Reich S, Zesiewicz T, Weiner WJ. Practice Parameter: neuroprotective strategies and alternative therapies for Parkinson disease (an evidence-based review): report of the Quality Standards Subcommittee of the American Academy of Neurology. Neurology. 2006;66(7):976-82. Epub 2006/04/12.

12. Kaski D, Quadir S, Patel M, Yousif N, Bronstein AM. Enhanced locomotor adaptation after-effect in the 'broken escalator' phenomenon using anodal tDCS. J

Neurophysiol. 2012. Epub 2012/02/11.

13. Toro BN, C.; Farren, P. A review of observational gait assessment in clinical practice. Physiotherapy theory and practice. 2003;19:137-49.

14. Lang A. Clinical rating scales and videotape analysis. In: Koller WC PG, editor. Therapy of Parkinson's disease. New York: Marcel Dekker; 1995. p. 21-46.

15. Allum JH, Carpenter MG. A speedy solution for balance and gait analysis: angular velocity measured at the centre of body mass. Curr Opin Neurol. 2005;18(1):15-21. Epub 2005/01/19.

16. Fahn S, Oakes D, Shoulson I, Kieburtz K, Rudolph A, Lang A, et al. Levodopa and the progression of Parkinson's disease. The New England journal of medicine.

2004;351(24):2498-508. Epub 2004/12/14.

17. Almeida QJ, Frank JS, Roy EA, Patla AE, Jog MS. Dopaminergic modulation of timing control and variability in the gait of Parkinson's disease. Mov Disord.

2007;22(12):1735-42. Epub 2007/06/09. 
18. Stefani A, Peppe A, Pierantozzi M, Galati S, Moschella V, Stanzione P, et al. Multitarget strategy for Parkinsonian patients: the role of deep brain stimulation in the centromedian-parafascicularis complex. Brain Res Bull. 2009;78(2-3):113-8. Epub 2008/09/25.

19. Khedr EM, Rothwell JC, Shawky OA, Ahmed MA, Hamdy A. Effect of daily repetitive transcranial magnetic stimulation on motor performance in Parkinson's disease. Mov Disord. 2006;21(12):2201-5. Epub 2007/01/16.

20. Lomarev MP, Kanchana S, Bara-Jimenez W, Iyer M, Wassermann EM, Hallett M. Placebo-controlled study of rTMS for the treatment of Parkinson's disease. Mov Disord. 2006;21(3):325-31. Epub 2005/10/08.

21. Benninger DH, Berman BD, Houdayer E, Pal N, Luckenbaugh DA, Schneider L, et al. Intermittent theta-burst transcranial magnetic stimulation for treatment of Parkinson disease. Neurology. 2011;76(7):601-9. Epub 2011/02/16.

22. Benninger DH, Lomarev M, Lopez G, Wassermann EM, Li X, Considine E, et al. Transcranial direct current stimulation for the treatment of Parkinson's disease. J Neurol Neurosurg Psychiatry. 2010;81(10):1105-11. Epub 2010/09/28.

23. Geroin C, Picelli A, Munari D, Waldner A, Tomelleri C, Smania N. Combined transcranial direct current stimulation and robot-assisted gait training in patients with chronic stroke: a preliminary comparison. Clin Rehabil. 2011;25(6):537-48. Epub 2011/03/16.

24. Jeffery DT, Norton JA, Roy FD, Gorassini MA. Effects of transcranial direct current stimulation on the excitability of the leg motor cortex. Experimental brain research Experimentelle Hirnforschung Experimentation cerebrale. 2007;182(2):281-7. Epub 2007/08/25.

\section{Figure legends}

Figure 1. Flow diagram showing enrolment and outcomes.

Figure 2. The pull test: representative traces and objective outcome measures, and correlation between pull test data and number of falls in the previous year.

(A) Representative traces for a patient (black line) and healthy age-matched control (grey line) for the pull test. Time taken to regain posture (duration to recovery) was calculated in seconds from the onset of the first negative displacement of the trunk pitch trace (small arrowhead) to arriving at +/- 2SD of the baseline pitch trace (large arrowhead). The peak recovery velocity $(\mathrm{Vp})$ within the first $100 \mathrm{~ms}$ after peak backward displacement was calculated from the differential of the position trace (inset). The main outcome measure was time (s) taken to regain posture following the retropulsive stimulus. Vertical bars represent standard errors. (B) Correlation between number of falls in the preceding year and the time (s) taken to regain posture following the retropulsive stimulus (objective measure for the pull test). Number of falls in the preceding year were calculated 
using patient's medical records, and questionnaire data. Data from the pull test are from the current study. * $\mathrm{p}<0.05$

Table 1. Comparison of different gait and balance measures between patients undergoing physical training and without physical training.

Table 2. Grouped data showing absolute values for gait velocity, stride length, Timed Up and Go duration, 6m walk duration and pull test results. 
Table 1. Comparison of the different gait and balance measures between patients undergoing physical training and without physical training

\begin{tabular}{llcccc}
\hline \multirow{2}{*}{$\begin{array}{l}\text { Group } \\
\text { Stimulation }\end{array}$} & Measure & \multicolumn{2}{c}{ Group I $(\mathrm{n}=8)$} & \multicolumn{2}{c}{ Group II $(\mathrm{n}=8)$} \\
\cline { 3 - 6 } Real $(\mathrm{n}=8)$ & \% Change & SD & \% Change & SD \\
& 6m Walk (s) & -18.89 & 15.1 & -4.29 & 10.9 \\
& Gait velocity (m/s) & -29.53 & 13.66 & -7.65 & 15.5 \\
& Stride length (m) & 54.21 & 17.16 & 13.02 & 11.2 \\
& TUG (s) & -45.3 & 29.3 & -10.89 & 8.8 \\
& Turn & -66.7 & 12.8 & -2.5 & 10.3 \\
& Retropulsion (s) & -50.89 & 36.5 & -1.44 & 31.5 \\
Sham (n=8) & & & & & \\
& 6m Walk (s) & -7.8 & 16.2 & -0.96 & 9.0 \\
& Gait velocity (m/s) & -15.51 & 12.3 & 2.91 & 10.1 \\
& Stride length (m) & 17.86 & 13.8 & 2.55 & 12.1 \\
& TUG (s) & -18.41 & 9.24 & -5.99 & 10.57 \\
& Turn & -15.3 & 8.9 & -4.5 & 10.6 \\
& Retropulsion (s) & -1.44 & 11.5 & -0.49 & 11.1 \\
\hline
\end{tabular}

Table 2. Grouped data showing absolute values for gait velocity, stride length, Timed Up and Go duration, $6 \mathrm{~m}$ walk duration and pull test results.

\begin{tabular}{|c|c|c|c|c|c|}
\hline \multirow{2}{*}{$\begin{array}{l}\text { Group } \\
\text { Stimulation }\end{array}$} & \multirow{2}{*}{ Measure } & \multicolumn{2}{|c|}{ Group I $(n=8)$} & \multicolumn{2}{|c|}{ Group II $(n=8)$} \\
\hline & & $\begin{array}{c}\text { Baseline } \\
\text { (SD) }\end{array}$ & $\begin{array}{c}\text { Final } \\
\text { (SD) }\end{array}$ & $\begin{array}{c}\text { Baseline } \\
\text { (SD) }\end{array}$ & $\begin{array}{r}\text { Final } \\
(\mathrm{SD})\end{array}$ \\
\hline \multicolumn{6}{|l|}{$\operatorname{Real}(n=8)$} \\
\hline & $6 \mathrm{~m}$ Walk (s) & $9.20(3.7)$ & $6.33(2.8)$ & $8.27(1.5)$ & $7.96(1.3)$ \\
\hline & Gait velocity $(\mathrm{m} / \mathrm{s})$ & $0.63(0.15)$ & $0.86(0.25)$ & $0.63(0.11)$ & $0.72(0.12)$ \\
\hline & Stride length $(\mathrm{m})$ & $90.0(18.9)$ & $127.3(17.7)$ & $90.9(16.1)$ & $95.2(16.1)$ \\
\hline & TUG (s) & $11.43(3.7)$ & $9.50(3.1)$ & $11.24(3.3)$ & $11.17(2.6)$ \\
\hline & Turn & $4.50(3.7)$ & $2.69(2.1)$ & $4.17(1.0)$ & $3.95(1.4)$ \\
\hline & Retropulsion (s) & $2.14(1.5)$ & $1.07(0.51)$ & $2.08(0.9)$ & $2.05(0.9)$ \\
\hline \multicolumn{6}{|l|}{ Sham $(n=8)$} \\
\hline & $6 \mathrm{~m}$ Walk (s) & $8.70(3.5)$ & $7.66(2.8)$ & $8.48(1.1)$ & $8.69(1.3)$ \\
\hline & Gait velocity $(\mathrm{m} / \mathrm{s})$ & $0.65(0.14)$ & $0.81(0.14)$ & $0.59(0.15)$ & $0.60(0.12)$ \\
\hline & Stride length $(\mathrm{m})$ & $91.4(21.7)$ & $120.7(22.6)$ & $89.4(17.6)$ & $90.6(16.1)$ \\
\hline & TUG (s) & $11.02(2.4)$ & $9.37(1.5)$ & $11.24(3.3)$ & $11.17(2.6)$ \\
\hline & Turn & $4.11(2.0)$ & 3.57 (1.9) & $4.14(1.2)$ & $4.00(1.1)$ \\
\hline & Retropulsion (s) & $2.07(1.2)$ & $1.51(0.8)$ & $2.01(1.2)$ & $2.00(1.0)$ \\
\hline
\end{tabular}




\section{Appendix 1. Exercise protocol for the physical training group.}

\section{Exercise Programme for PD}

\section{Stand up from a sitting position}

Follow a sequence of four actions:

- Shifting the body forward so that the buttocks are close to the edge of the chair,

- Placing the feet flat on the floor so that the heels are well back,

- Leaning the trunk forward, and

- Standing up quickly while thinking of leaning "forward and up" in an arc of movement.

Note: It is useful to start with a chair with a high seat and armrests so the sit-tostand action is performed independently and to gradually reduce seat height (and presence of armrests).

\section{Walking}

Walk with long strides and adequate ground clearance in different walking speed

- Initially, draw some lines on the floor and ask the patient to walk by following the lines with large steps

- Give the patient a rhythmical beat to generate a more normal stepping rate

- Ask the patient to walk with swinging the arms to help balancing

Walk with long strides and take a side step at the end

Step over obstacles while walking

Note: Remind the patient to stand upright and look forward while walking

\section{Turning}

Turn in a large arc of movement by using full body movements rather than focusing on rapidly switching directions

- Put an object, such as a chair, in the centre of turning and ask the patient to turn by taking steps around the object

In very small spaces, where turning in a large arc is not possible, the "clock turn" strategy is recommended

Note: Attention is directed to lifting the feet clear in a deliberate stepping action, rather than shuffling or swivelling around

\section{Balance training}

Forward reach a target with both hands to different directions, from closer to further distance

Pick up some objects from the floor (or a lower place) to a higher place 